\title{
RUMO À OTIMIZAÇÃO DE ESTRATÉGIAS DE MANEJO DE ARBOVIROSES: POTENCIAL BIOINSETICIDA DE EXTRATOS VEGETAIS CONTRA AEDES SPP. (DIPTERA: CULICIDAE)
}

\author{
TOWARDS THE OPTIMIZATION OF ARBOVIRUSES MANAGEMENT \\ STRATEGIES: BIOINSECTICIDE POTENTIAL OF PLANT EXTRACTS \\ AGAINST AEDES SPP. (DIPTERA: CULICIDAE)
}

\author{
Giulian César da Silva Sá ${ }^{1}$ \\ Laísa Vilar Cordeiro ${ }^{2}$ \\ Pedro Thiago Ramalho de Figueiredo ${ }^{3}$ \\ Thamara Rodrigues de Melo ${ }^{4}$ \\ Aleson Pereira de Sousa ${ }^{5}$
}

RESUMO: Mosquitos medicamente desafiadores do gênero Aedes são vetores de potenciais patógenos de doenças que assolam populações ao redor do mundo, como dengue, febre amarela, zika e febre chikungunya. Das estratégias de controle comumente disponíveis, tem-se os pesticidas sintéticos. Porém, efeitos colaterais ao ecossistema e aos organismos não-alvo são perceptíveis e seu uso excessivo contribuiu para o desenvolvimento de resistência nos mosquitos. Reconhecendo a relevância dessa problemática, a presente pesquisa objetivou apresentar uma revisão sistemática que abordasse a necessidade imediata do desenvolver novas medidas de controle de vetores, apontando os inseticidas botânicos como escolha ecologicamente mais viável. Das investigações realizadas, demarcou-se 554

\footnotetext{
1 Biólogo, Especialista em Ciências Ambientais, Mestre em Biologia Celular e Molecular, e Doutorando em Bioquímica (UFRN). Pesquisador do Instituto de Medicina Tropical do Rio Grande do Norte. E-mail: giuliancesarsa@gmail.com.

2 Farmacêutica, Especialista em Atenção Farmacêutica e Farmácia Clínica, Mestre em Biologia Celular e Molecular, e Doutoranda em Produtos Naturais e Sintéticos Bioativos (área de concentração: Farmacologia) (UFPB). E-mail: laisavilar@gmail.com.

3 Farmacêutico, Mestre e Doutorando em Produtos Naturais e Sintéticos Bioativos (área de concentração: Farmacologia) (UFPB). E-mail: pedrotrfigueiredo@hotmail.com.

${ }^{4}$ Farmacêutica, Especialista em Análises Clínicas, Mestre em Ciências Farmacêuticas, e Doutoranda em Produtos Naturais e Sintéticos Bioativos (área de concentração: Farmacologia) (UFPB). E-mail: th.rmelo@outlook.com.

${ }^{5}$ Biomédico, Especialista em Citologia Clínica, Mestre em Biologia Celular e Molecular, e Doutorando em Desenvolvimento e Inovação Tecnológica de Medicamento (UFPB). E-mail: aleson_155@hotmail.com.
} 
estudos que, após emprego do processo de triagem e seleção, perfizeram 20 artigos; conforme critérios previstos para essa revisão. Tais estudos apontam que, diferente dos pesticidas sintéticos, os inseticidas botânicos, por serem obtidos de misturas complexas, são capazes de comprometer os processos fisiológicos e comportamentais do mosquito, afetando negativamente a absorção e o armazenamento de nutrientes, bem como a metamorfose e a fertilidade do adulto, sem lhes gerar mecanismos de resistência. Contudo, mesmo apresentando tantos benefícios ou sendo biodegradáveis e obtidos de fontes seguras, o número diminuto de artigos encontrados em nossas buscas reforça a necessidade de intensificar as pesquisas sobre uso de extratos vegetais no combate de Aedes spp.

Palavras chave: Aedes. Extratos Vegetais. Infecções por Arbovírus. Controle de Vetores. Culicidae.

ABSTRACT: Medically challenging mosquitoes of the Aedes genus are vectors of potential pathogens of diseases that concern populations around the world, such as dengue, yellow fever, zika and chikungunya fever. Synthetic pesticides are the control strategies commonly available, but side effects to the ecosystem and non-target organisms are noticeable and their overuse has contributed to the development of resistance in mosquitoes. Recognizing the relevance of this problem, the present research aimed to present a systematic review on the immediate need for the development of new vector control measures, with botanical insecticides being the ecologically first choice. 554 studies were demarcated and, after using the process of screening and selection of studies, 20 articles were selected; according to the criteria proposed for this review. Studies suggest that, unlike synthetic pesticides, botanical insecticides, because they are obtained from complex mixtures, are capable of compromising the mosquitoes' physiological and behavioral processes, negatively affecting the absorption and storage of nutrients, as well as metamorphosis and fertility adult, without generating resistance mechanisms in mosquitoes. However, even with so many benefits or being safe and biodegradable sources, the small number of articles found in our searches reinforces the need to intensify research on the use of plant extracts to combat of Aedes spp.

Keywords: Aedes. Plant Extracts. Arbovirus Infections. Vector Control. Culicidae. 


\section{INTRODUÇÃO}

Espécies de mosquitos medicamente desafiadoras dos gêneros Anopheles, Aedes e Culex são vetores de potenciais patógenos de doenças que assolam populações ao redor do mundo, causando milhares de mortes por ano (KARTHI et al., 2020; AZIZ et al., 2019; SUKKANON et al., 2019; RAVI et al., 2018). A década passada, particularmente, presenciou um aumento exponencial na incidência de doenças transmitidas por mosquitos (SHARMA, TILAK \& SISODIA, 2019), onde esse cenário acabou se tornando uma questão desafiadora para a saúde pública, especialmente em países tropicais e subtropicais, e seus malefícios são vistos até hoje (PRATHEEBA et al., 2019; YADAV et al., 2019). Os programas e estratégias de controle vêm enfrentando desafios importantes, incluindo surtos de novos arbovírus, desenvolvimento de mecanismos de resistência nos mosquitos, além da rápida e crescente disseminação de mosquitos altamente invasivos nos mais diversos ecossistemas (ALI, GOPALAKRISHNAN \& VENKATESALU, 2018).

Os mosquitos da ordem Diptera, em especial da família Culicidae, são capazes de transmitir doenças humanas letais, como encefalite japonesa, malária, dengue, febre amarela, filariose linfática e febre do Nilo Ocidental (KUMAR, KUMAR \& AGRAWAL, 2018; THANIGAIVEL et al., 2018). Dentre todas essas, a dengue é a infecção arboviral humana mais prevalente, cujo vírus é transmitido principalmente por mosquitos fêmeas do gênero Aedes (PRATHEEBA et al., 2019; PILAQUINGA et al., 2019; KAMARAJ et al., 2018; HARI \& MATHEW, 2018; RODRIGUES NETO et al., 2018) e sua ocorrência global foi ampliada recentemente (AZIZ et al., 2019), podendo ainda transmitir o vírus da Zika e febre Chikungunya (SILVA et al., 2019; SHARMA, TILAK \& SISODIA, 2019; CHELLAPPANDIAN et al., 2019).

A febre Zika tem ganhado maior ênfase por sua associação com má formação congênita e microcefalia, mas todas essas arboviroses apresentam alto risco de disseminação, morbidade e mortalidade humana em muitos países (SÁ et al., 2020), pois ainda não há vacina para prevenir a infecção de todas elas e nem drogas 
específicas para combater os vírus (PRATHEEBA et al., 2019), sendo o controle do vetor a solução comumente viável (SÁ et al., 2020; SOGAN et al., 2018; KUMAR, KUMAR \& AGRAWAL, 2018).

Das estratégias de controle disponíveis, acredita-se que as populações de Aedes em áreas urbanas e periurbanas podem ser significativamente suprimidas com uso de inseticidas, não sendo vista a mesma eficácia quando destinadas às populações de ambientes rurais (KARTHI et al., 2020). Tradicionalmente, empregase inseticidas sintéticos, incluindo organoclorados, organofosforados, carbamatos e piretróides, porém efeitos colaterais ao ecossistema e aos organismos não-alvo, incluindo seres humanos, são perceptíveis e seu uso excessivo contribui para o desenvolvimento de resistência nos mosquitos (AZIZ et al., 2019; PILAQUINGA et al., 2019; INOCENTE et al., 2019; CHELLAPPANDIAN et al., 2019).

Sendo também possível demarcar o uso de abordagens mecânicas (eliminação ou proteção dos locais de reprodução) e biológicas (emprego de espécies predadoras ou entomopatogênicas) em seu controle (SÁ et al., 2020).

No Brasil, muitas foram as estratégias utilizadas para o contingenciamento das ações de Aedes spp. como fruto das intervenções originadas em 1947, quando por intermédio do Programa de Erradicação de Aedes aegypti no Hemisfério Oeste, a Organização Pan-Americana da Saúde e a Organização Mundial da Saúde decidiram coordenar sua erradicação no continente. Entretanto, um ano depois, foi confirmada a reintrodução do mosquito no Brasil, que não conseguiu ser contido por combate químico, possibilitando novamente sua dispersão, o que impulsionou o surgimento de programas de erradicação do mosquito, inicialmente coordenado pela Superintendência de Campanhas de Saúde Pública (SUCAM), por intermédio do Programa Nacional de Controle da Febre Amarela e Dengue. Mesmo sem tanto êxito, as autoridades nacionais competentes continuaram a direcionar esforços para sua erradicação. Décadas depois, surgem armadilhas capazes de capturar fêmeas grávidas e adultas de $A$. aegypti, sendo duas armadilhas produzidas: Adultrap e MosquiTrap (SÁ et al., 2020).

Embora exista essa pressão histórica envolvendo o uso de inseticidas sintéticos pelos programas de controle de vetores, os índices de arboviroses continuam a preocupar populações ao redor do globo, os casos de resistência nos 
vetores têm crescido e novas doenças transmitidas por mosquitos têm surgido. Ademais, Thanigaivel et al. (2018) apontam que aplicações repetidas de inseticidas sintéticos levam a efeitos adversos em mosquitos benéficos, como polinizadores e predadores, desequilibrando o fluxo natural dos ecossistemas. Com isso, pesquisas (INOCENTE et al., 2019; FALKOWSKI et al., 2019; SILVA et al., 2019; KAMARAJ et al., 2018) têm apontado como necessidade imediata o desenvolvimento de novas medidas de controle de vetores isentas desses problemas, sendo os inseticidas botânicos a escolha ecologicamente mais apropriada.

Os inseticidas botânicos tornam-se uma possibilidade de uso no combate de Aedes spp. por dispor de uma rica, complexa e segura fonte de componentes bioativos e biodegradáveis (PRATHEEBA et al., 2019; CHELLAPPANDIAN et al., 2018; RAVI et al., 2018; KAMARAJ et al., 2018), que podem atuar sinergicamente em diferentes alvos moleculares dentro do mosquito, reduzindo os índices de resistência devido às suas estruturas complexas (PILAQUINGA et al., 2019; YADAV et al., 2019; THANIGAIVEL et al., 2018) e ser rastreados para atividades bioinseticidas (INOCENTE et al., 2019; SOGAN et al., 2018; ALI, GOPALAKRISHNAN \& VENKATESALU, 2018). Assim, as consideráveis necessidades, desafios e potencialidades desta pesquisa são descritos em uma revisão sistemática, apresentando como os inseticidas botânicos podem fornecer ferramentas eficazes e ecologicamente corretas no combate a populações de Aedes.

\section{METODOLOGIA}

O presente estudo baseou-se nas diretrizes metodológicas para realização de revisão sistemática, buscando-se analisar a eficácia de extratos vegetais como uma alternativa ao controle de populações de Aedes, com vistas ao terceiro objetivo da Agenda 2030, que visa assegurar uma vida saudável e promover o bem-estar para todos, em todas as idades. As metas norteadoras para esta pesquisa foram a $3.3 \mathrm{e}$ 3.d da Agenda 2030, que concernem, respectivamente, em controlar as doenças tropicais negligenciadas e combater outras mazelas até 2030; e reforçar a 
capacidade de todos os países, particularmente aqueles em desenvolvimento, para o alerta precoce, redução de riscos e gerenciamento de riscos nacionais e globais à saúde.

A evidência de investigação foi demarcada mediante busca eletrônica no banco de dados virtual PubMed (National Library of Medicine National Institutes of Health) com uso dos descritores de assunto MeSH (Medical Subject Headings), empregando o operador lógico booleano "AND".

Os parâmetros de elegibilidade que complementaram a questão de pesquisa tiveram como critérios de inclusão a adequação e análise refinada dos estudos (full text) que versassem sobre a utilização de extratos vegetais no combate ao mosquito vetor de inúmeras arboviroses (Aedes spp.); período mínimo de seguimento de análise (estudos publicados entre 01 de janeiro de 2018 até 31 de dezembro de 2020); e Inglês como idioma padrão. Já os critérios de exclusão envolveram a não aceitação de dados não publicados ou em andamento, literatura cinzenta, artigos de revisão e pesquisas puramente etnobotânicas e etnofarmacológicas.

O processo de triagem e seleção dos estudos deu-se pelo emprego das diretrizes dos Itens de Relatórios Preferidos para Revisões Sistemáticas e MetaAnálises (PRISMA), realizado pelos autores de forma independente. Todos os estudos triados tiveram sua elegibilidade confirmada pela leitura do texto completo, cuja razão primária da exclusão contemplava aqueles estudos cuja metodologia e resultados não estivessem adequados ao fluxo da pesquisa, de modo que sua questão de pesquisa não respondesse às análises, mediante leitura inicial de título e resumo.

Por fim, a fase de interpretação dos resultados, visou identificar lacunas existentes na literatura no que tange o manejo de arboviroses e apontar estratégias ecologicamente amigáveis, permitindo destacar sugestões pertinentes para futuras pesquisas direcionadas para a melhoria da assistência à saúde, mediante análise do artigo em sua íntegra. 


\section{RESULTADOS E DISCUSSÃO}

Das investigações realizadas, demarcou-se 554 estudos que, após emprego do processo de triagem e seleção, perfizeram 59 artigos. Ao passo em que as buscas eram refinadas e os estudos triados tiveram sua elegibilidade confirmada pela leitura do texto completo, esse número totalizou 20 artigos; conforme critérios previstos para essa revisão (Figura 1). Mesmo apresentando tantos benefícios ou sendo fontes seguras e biodegradáveis (PRATHEEBA et al., 2019; CHELLAPPANDIAN et al., 2018), o número diminuto de artigos encontrados em nossas buscas, após aplicação dos filtros, reforça a necessidade de intensificar as pesquisas sobre uso de extratos vegetais no combate a populações de Aedes. 
Figura 1 - Fluxograma da estratégia de identificação, critérios de elegibilidade e seleção dos potenciais estudos.
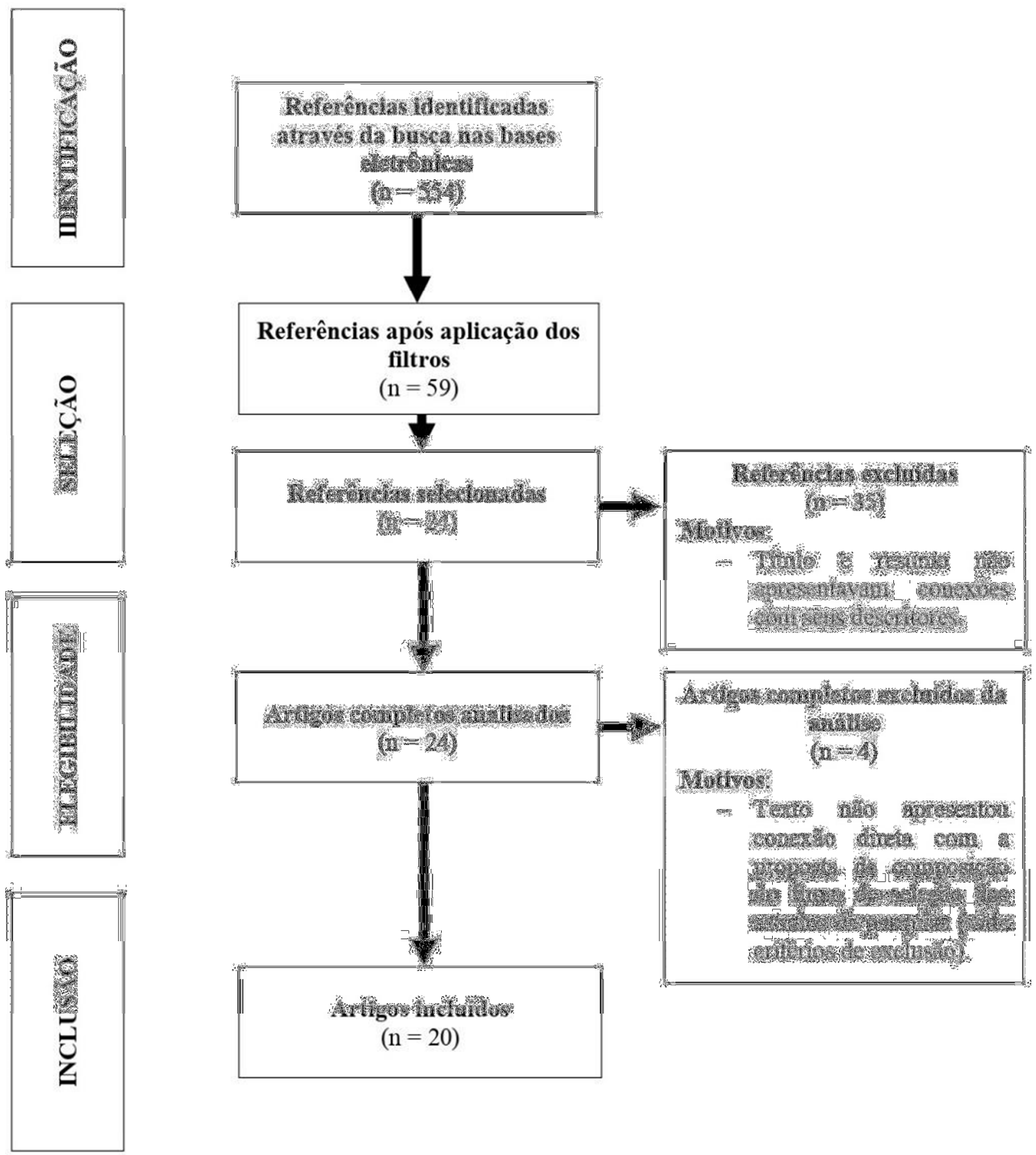

A Tabela 1 apresenta os estudos elegíveis pelas buscas realizadas, apontando uma gama de espécies vegetais de 21 famílias botânicas diferentes com potencial uso como bioinseticida. Dentre as famílias botânicas listadas, 
Acanthaceae, Apocynaceae, Asteraceae e Piperaceae foram as que apresentaram o maior número de espécies com estudos realizados, sendo possível demarcar diversos sistemas extratores, tanto aquosos quanto alcoólicos, bem como a diversa gama de partes vegetais passíveis de serem exploradas.

Tabela 1 - Relação dos estudos elegíveis pelas buscas realizadas.

Referência

Ali, Gopalakrishnan \& Venkatesalu (2018)

Ali, Gopalakrishnan \& Venkatesalu (2019)

Benelli et al. (2018)

Chellappandian et al. (2019)

Falkowski et al. (2019)

Hari \& Mathew (2018)

$\mathrm{ME}, \mathrm{CL}$ e EP

Kamaraj et al. (2018)

Karthi et al. (2020)

Kumar, Kumar \&

Agrawal (2018)

Macedo et al. (2018)

Pineda-Cortel et al.

(2019)

Pratheeba et al.

(2019)

Ravi et al. (2018)

Rodrigues Neto et al. (2018)

Sharma, Tilak \&

Sisodia (2019)

Silva et al. (2019)

Sogan et al. (2018)
Solução extratora

EP, CL, EA e ME

ME

AG

ET

EA, ME, EP e AG $C L$ e AG $\mathrm{HE}$

$\mathrm{HE}, \mathrm{EA}$ e $\mathrm{AG}$

$\mathrm{HE}, \mathrm{EA}, \mathrm{CL}$, $A C$ e $M E$

ME

AG

AG

AG

ME
Cichorium intybus (Asteraceae) e Artemisia absinthium (Asteraceae) // raízes e partes aéreas

Juglans regia (Juglandaceae) // flores

Aglaia elaeagnoidea (Meliaceae) // folhas Trichodesma indicum (Boraginaceae) // folhas

85 espécies diferentes, 36 famílias // todas as partes do vegetal

Hyptis suaveolens (Lamiaceae), Lantana camara (Verbenaceae), Nerium oleander

(Apocynaceae) e Tecoma stans (Bignoniaceae) // folhas

Adiantum caudatum (Adiantaceae), Actiniopteris radiata (Actiniopteridaceae), Lycopodium clavatum (Lycopodiaceae) e Hemionitis arifolia (Hemionitidaceae) // folhas

$\mathrm{AC}, \mathrm{ME}, \mathrm{AE}, \quad$ Avicennia marina (Acanthaceae) // folhas

ME, HE, AC, Holarrhena antidysenterica (Apocynaceae)
// caules

Piper solmsianum (Piperaceae) // folhas

Artocarpus blancoi (Moraceae) // folhas

Pavetta tomentosa e Tarenna asiática

(Rubiaceae) // folhas

Azolla pinnata (Salviniaceae) // corpo vegetativo inteiro

Rhizophora mangle (Rhizophoraceae) // folhas

Bougainvillea spectabilis (Nyctaginaceae), Saraca asoca (Fabaceae) e Chenopodium álbum (Chenopodiaceae) // flores e folhas Ricinus communis (Euphorbiaceae) // Moringa oleifera (Moringaceae) // sementes 
Sukkanon et al.

(2019)

Thanigaivel et al.

(2018)

Yadav et al. (2019)
ET

ME

folhas e sementes Andrographis paniculata (Acanthaceae) // folhas Alangium salvifolium (Alangiaceae) // folhas

$C L, E A, H E$, ME e AG

Legenda: $\mathrm{AC}$, acetona; $\mathrm{ME}$, metanol; $\mathrm{EA}$, acetato de etila; $\mathrm{BP}$, benzeno de petróleo; $\mathrm{HE}$, hexano; CL, clorofórmio; ET, etanol; EP, éter de petróleo; e AG, água.

Notoriamente, a excepcional biodiversidade de plantas impulsiona o despertar para sua promissora quimiodiversidade, em função de suas interações dinâmicas com o ambiente, e por amparar o sistema de defesa vegetal contra predadores e pragas durante a sua evolução (FALKOWSKI et al., 2019; SILVA et al., 2019). Ademais, diferente dos inseticidas sintéticos, aqueles feitos à base de extratos vegetais, por serem obtidos de misturas complexas, são capazes de comprometer os processos fisiológicos e comportamentais do mosquito, sem lhes gerar mecanismos de resistência (RAVI et al., 2018). O tipo de defesa também é peculiar à espécie vegetal estudada, onde espécies de vida longa e crescimento lento, alocam recursos para defesa em quantidades altamente concentradas, enquanto espécies de vida curta, sintetizam menor quantidade de compostos tóxicos (FALKOWSKI et al., 2019).

O subgênero Stegomyia do gênero Aedes contém aproximadamente 110 espécies cosmotropicais descritas que habitam ambientes urbanos, principalmente no intra e peridomicílio humano, sendo difícil de ser encontrados em ambientes semisilvestres; procriam tanto em criadouros naturais ou artificiais, que acumulam água em períodos chuvosos; apresentam desenvolvimento holometábolo, organizado em quatro estágios distintos: ovicida, larvicida, pupicida e adulticida, que podem se completar entre 1,5 a 3 semanas; e o ciclo de vida do mosquito constituise de duas fases: uma terrestre, onde os adultos vivem, e uma aquática, onde ocorre a evolução de todas as formas imaturas. Tanto machos e fêmeas adultos dependem da ingestão de carboidratos. Porém, adicionalmente, as fêmeas apresentam hábito alimentar hematófago, preferencialmente de sangue humano, para obtenção de ferro e posterior maturação dos ovários (SÁ et al., 2020).

O intestino médio de Aedes aegypti é composto tanto por células epiteliais digestivas (colunares) quanto estaminais (regenerativas). Quando os compostos 
presentes nos extratos vegetais adentram nos intestinos do Aedes, eles comprometem o funcionamento dessa estrutura, afetando negativamente a absorção e o armazenamento de nutrientes, bem como a metamorfose e a fertilidade do adulto (SILVA et al., 2019). Entretanto, as propriedades antinutricionais desses extratos variam com a idade da planta, espécie, parte extraída, local de coleta e solvente usado para extração (HARI \& MATHEW, 2018). Isso comprova a variedade de LC50 (concentração capaz de ser letal para $50 \%$ dos insetos testados) observada nos estudos encontrados nessa pesquisa.

É necessária também uma análise sobre os aspectos históricos e da distribuição geográfica desses vetores, detendo-se a correlacionar os locais de incidência das arboviroses com a chegada dos mosquitos, em especial, ao cenário brasileiro. Paralelamente, estudos envolvendo a bioecologia e o ciclo de vida de Aedes spp. também são essenciais para melhor compreensão de seu desenvolvimento e possibilita, a partir disso, elaborar estratégias eficazes para o controle, auxiliando na redução de surtos de doenças por eles causadas (SÁ et al., 2020).

\section{CONSIDERAÇÕES FINAIS}

O controle de populações de Aedes envolve comumente o emprego de inseticidas químicos que atuam no Sistema Nervoso Central dos mosquitos. Contudo, é notório o desenvolvimento de resistência nos mosquitos por seu uso intensivo e prolongado, além de acarretar problemas ambientais e na saúde de espécies não-alvo, resultando no fracasso do controle de vetor e demandando a busca por novas e eficazes estratégias. Apontamos como possibilidade o uso de extratos botânicos como ferramentas promissoras para o controle de Aedes spp., face a acessibilidade da matéria-prima (vegetais), baixa toxicidade e pouca possibilidade de desencadeamento de mecanismos de resistência por seus compostos atuarem em diferentes sítios de ação no mosquito. 
Entretanto, nenhuma dessas ferramentas terá sucesso caso a população não participe dos processos de sua implementação e colabore com as estratégias de manejo já existentes e que poderão vir a suplantar o atual cenário, levando a erradicação das arboviroses.

Acreditamos, portanto, que a conquista dos objetivos para o desenvolvimento sustentável da nação é possível e que as doenças negligenciadas, em especial arboviroses, podem ser reparadas através da incorporação e utilização de novas tecnologias voltadas à promoção de saúde, valendo-se das teses: 1) a política atual do Ministério da Saúde instiga o estímulo ao desenvolvimento e inovação de tecnologias diagnósticas, terapêuticas e preventivas na melhoria da saúde dos brasileiros; e 2) a implementação dessas formas de controle acompanham a expansão da melhoria do acesso e qualidade da atenção nos sistemas de saúde, reduzindo os impactos ambientais e aumentando os impactos desejados sobre a saúde e qualidade de vida da população.

\section{AGRADECIMENTOS}

Os autores agradecem ao Conselho Nacional de Desenvolvimento Científico e Tecnológico (CNPq), à Coordenação de Aperfeiçoamento de Pessoal de Nível Superior (CAPES) e ao Ministério da Ciência, Tecnologia, Inovação e Comunicações (MCTIC) pelo apoio financeiro. Os autores gostariam de agradecer ainda ao Instituto de Medicina Tropical do Rio Grande do Norte, a Universidade Federal do Rio Grande do Norte e a Universidade Federal da Paraíba, além dos pesquisadores e Programas de Pós-graduações destas instituições. 


\section{REFERÊNCIAS BIBLIOGRÁFICAS}

Ali, S.I.; Gopalakrishnan, B.; Venkatesalu, V. Chicory (Cichorium intybus) and wormwood (Artemisia absinthium) extracts exhibit strong larvicidal activity against mosquito vectors of malaria, dengue fever, and filariasis. Parasitology International, 67(6):781-786, 2018. https://doi:10.1016/j.parint.2018.08.005.

Ali, S.I.; Gopalakrishnan, B.; Venkatesalu, V. Larvicidal potential of Juglans regia male flower against Anopheles stephensi, Aedes aegypti and Culex quinquefasciatus. Natural Product Research, 33(10):1463-1466, 2019. https://doi:10.1080/14786419.2017.1416374.

Aziz, A.T.; Alshehri, M.A.; Alanazi, N.A.; Panneerselvam, C.; Trivedi, S.; Maggi, F.; Sut, S.; Dall'Acqua, S. Phytochemical analysis of Rhazya stricta extract and its use in fabrication of silver nanoparticles effective against mosquito vectors and microbial pathogens. Science of The Total Environment, 15;700: 1-45. https://doi:10.1016/j.scitotenv.2019.134443.

Benelli, G.; Govindarajan, M.; Senthilmurugan, S.; Vijayan, P.; Kadaikunnan, S.; Alharbi, N.S.; Khaled, J.M. Fabrication of highly effective mosquito nanolarvicides using an Asian plant of ethno-pharmacological interest, Priyangu (Aglaia elaeagnoidea): toxicity on non- target mosquito natural enemies. Environmental Science and Pollution Research, 25(11):10283-10293, 2018. https://doi:10.1007/s11356-017-8898-4.

Chellappandian, M.; Senthil-Nathan, S.; Vasantha-Srinivasan, P.; Karthi, S.; Thanigaivel, A.; Kalaivani, K.; Sivanesh, H.; Stanley-Raja, V.; Chanthini, K.M.; Shyam-Sundar, N. Target and non-target botanical pesticides effect of Trichodesma indicum (Linn) R. Br. and their chemical derivatives against the dengue vector, Aedes aegypti L. Environmental Science and Pollution Research, 26(16):16303-16315, 2019. https://doi:10.1007/s11356-019-04870-3.

Falkowski, M.; Jahn-Oyac, A.; Odonne, G.; Flora, C.; Estevez, Y.; Touré, S.; Boulogne, I.; Robinson, J.C.; Béreau, D.; Petit, P.; Azam, D.; Coke, M.; Issaly, J.; Gaborit, P.; Stien, D.; Eparvier, V.; Dusfour, I.; Houël, E. Towards the optimization of botanical insecticides research: Aedes aegypti larvicidal natural products in French Guiana. Acta Tropica, 201:1- 18, 2019. https://doi:10.1016/j.actatropica.2019.105179.

Hari, I.; Mathew, N. Larvicidal activity of selected plant extracts and their combination against the mosquito vectors Culex quinquefasciatus and Aedes aegypti. Environmental Science and Pollution Research, 25(9):9176-9185, 2018. https://doi:10.1007/s11356-018- 1515-3.

Inocente, E.A.; Nguyen, B.; Manwill, P.K.; Benatrehina, A.; Kweka, E.; Wu, S.; Cheng, X.; Rakotondraibe, L.H.; Piermarini, P.M. Insecticidal and antifeedant activities of Malagasy medicinal plant (Cinnamosma sp.) extracts and drimane-type sesquiterpenes against Aedes aegypti mosquitoes. Insects, 10(11), 373, 2019. https://doi:10.3390/insects10110373.

Inocente, E.A.; Shaya, M.; Acosta, N.; Rakotondraibe, L.H.; Piermarini, P.M. A natural agonist of mosquito TRPA1 from the medicinal plant Cinnamosma fragrans that is toxic, antifeedant, and repellent to the yellow fever mosquito Aedes aegypti. PLOS Neglected Tropical Diseases, 12(2):1-19, 2018. https://doi:10.1371/journal.pntd.0006265.

Kamaraj, C.; Deepak, P.; Balasubramani, G.; Karthi, S.; Arul, D.; Aiswarya, D.; Amutha, V.; Vimalkumar, E.; Mathivanan, D.; Suseem, S.R.; Muthu-Pandian, C.K.; Senthil-Nathan, S.; Perumal, P. Target and non-target toxicity of fern extracts against mosquito vectors and beneficial aquatic organisms. Ecotoxicology and Environmental Safety, 161:221-230, 2018. https://doi:10.1016/j.ecoenv.2018.05.062. 
Karthi, S.; Vinothkumar, M.; Karthic, U.; Manigandan, V.; Saravanan, R.; Vasantha- Srinivasan, P.; Kamaraj, C.; Shivakumar, M.S.; De Mandal, S.; Velusamy, A.; Krutmuang, P.; SenthilNathan, S. Biological effects of Avicennia marina (Forssk.) vierh. extracts on physiological, biochemical, and antimicrobial activities against three challenging mosquito vectors and microbial pathogens. Environmental Science and Pollution Research, 2020. https://doi.org/10.1007/s11356-020-08055-1.

Kumar, D.; Kumar, G.; Agrawal, V. Green synthesis of silver nanoparticles using Holarrhena antidysenterica (L.) Wall.bark extract and their larvicidal activity against dengue and filariasis vectors. Parasitology Research, 117(2):377-389, 2018. https://doi.org/10.1007/s00436-0175711-8.

Macedo, A.L.; Duprat, R.C.; Moreira, D.L.; Kaplan, M.A.C.; Vasconcelos, T.R.A.; Pinto, L.C.; Montenegro, R.C.; Ratcliffe, N.A.; Mello, C.B.; Valverde, A.L. Isolation of a larvicidal compound from Piper solmsianum C.DC. (Piperaceae). Natural Product Research, 32(22):2701-2704, 2018. https://doi:10.1080/14786419.2017.1374265.

Pilaquinga, F.; Morejón, B.; Ganchala, D.; Morey, J.; Piña, N.; Debut, A.; Neira, M. Green synthesis of silver nanoparticles using Solanum mammosum L. (Solanaceae) fruit extract and their larvicidal activity against Aedes aegypti L. (Diptera: Culicidae). PLoS One, 14(10):1-13, 2019. https://doi:10.1371/journal.pone.0224109.

Pineda-Cortel, M.R.B.; Cabantog, R.J.R.; Caasi, P.M.; Ching, C.A.D.; Perez, J.B.S.; Godisan, P.G.M.; Latorre, C.M.G.; Lucero, D.R.; Salonga, R.B. Larvicidal and ovicidal activities of Artocarpus blancoi extracts against Aedes aegypti. Pharmaceutical Biology, 57(1):120-124, 2019. https://doi:10.1080/13880209.2018.1561727.

Pratheeba, T.; Taranath, V.; Sai Gopal, D.; Natarajan, D. Antidengue potential of leaf extracts of Pavetta tomentosa and Tarenna asiatica (Rubiaceae) against dengue virus and its vector Aedes aegypti (Diptera: Culicidae). Heliyon, 5(11):1-10, https://doi:10.1016/j.heliyon.2019.e02732.

Ravi, R.; Zulkrnin, N.S.H.; Rozhan, N.N.; Nik Yusoff, N.R.; Mat Rasat, M.S.; Ahmad, M.I.; Ishak, I.H.; Amin, M.F.M. Chemical composition and larvicidal activities of Azolla pinnata extracts against Aedes (Diptera: Culicidae). PLoS One, 6;13(11):1-18, 2018. https://doi:10.1371/journal.pone.0206982.

Rodrigues Neto, A.A.; Gomes Júnior, P.P.; Silva, M.C.; Lima, C.S.A.; Yara, R.; Guimarães, E.B.; Santana, E.S.; Silva, L.A.D.; Lira, E.J.R.V.; Vieira, J.R.C. Evaluation of embryotoxic and embryostatic effects of the aqueous extract of Rhizophora mangle and tannic acid on eggs and larvae of Aedes aegypti. Annals of the Brazilian Academy of Sciences, 90(2 suppl 1):21412148, 2018. https://doi.org/10.1590/0001-3765201720170297.

Sá, G.C.S.; Figueiredo, P.T.R.; Cordeiro, L.V.; Melo, T.R. Dos mecanismos de resistência a inseticidas aos avanços e desafios no manejo de epidemias de arboviroses, p.305-324. In: One, G.M.C. Meio Ambiente: tecnologia e sustentabilidade a serviço da vida. IMEA: 2020, 699p.

Sharma, A.; Tilak, R.; Sisodia, N. Evaluation of bioactivity of aqueous extracts of Bougainvillea spectabilis, Saraca asoca, and Chenopodium album against immature forms of Aedes aegypti. Medical Journal Armed Forces India, 75(3):308-311, 2019. https://doi:10.1016/j.mjafi.2018.07.013.

Silva, L.L.S.; Fernandes, K.M.; Miranda, F.R.; Silva, S.C.C.; Coelho, L.C.B.B.; Navarro, D.M.D.A.F.; Napoleão, T.H.; Martins, G.F.; Paiva, P.M.G. Exposure of mosquito (Aedes aegypti) larvae to the water extract and lectin-rich fraction of Moringa oleifera seeds impairs their development and future fecundity. Ecotoxicology and Environmental Safety, 183:1-7, 2019. https://doi:10.1016/j.ecoenv.2019.109583. 
Sogan, N.; Kapoor, N.; Singh, H.; Kala, S.; Nayak, A.; Nagpal, B.N. Larvicidal activity of Ricinus communis extract against mosquitoes. Journal of Vector Borne Disease, 55(4):282- 290, 2018. https://doi:10.4103/0972-9062.256563.

Sukkanon, C.; Karpkird, T.; Saeung, M.; Leepasert, T.; Panthawong, A.; Suwonkerd, W.; Bangs, M.J.; Chareonviriyaphap, T. Excito-repellency Activity of Andrographis paniculata (Lamiales: Acanthaceae) against colonized mosquitoes. Journal of Medical Entomology, 9;57(1):192203, 2019. https://doi:10.1093/jme/tjz139.

Thanigaivel, A.; Vasantha-Srinivasan, P.; Edwin, E.S.; Ponsankar, A.; Selin-Rani, S.; Chellappandian, M.; Kalaivani, K.; Senthil-Nathan, S.; Benelli, G. Development of an ecofriendly mosquitocidal agent from Alangium salvifolium against the dengue vector Aedes aegypti and its biosafety on the aquatic predator. Environmental Science and Pollution Research, 25(11):10340-10352, 2018. https://doi:10.1007/s11356-017-9102-6 Yadav, R.; Saini, H.; Kumar, D.; Pasi, S.; Agrawal, V. Bioengineering of Piper longum L. extract mediated silver nanoparticles and their potential biomedical applications. Materials Science \& Engineering C, 104:1-17, 2019. https://doi:10.1016/j.msec.2019.109984. 